\title{
Utilisation de l'imagerie vidéo pour la gestion intégrée des côtes : application à la gestion touristique de la plage du «Puntal» à Santander (Espagne)
}

\author{
Andres F. Osorio ${ }^{1,2}$, Raúl Medina ${ }^{2}$, Nicolas Garcia ${ }^{3}$, Marc Labégorre ${ }^{4}$ \\ ${ }^{1}$ Escuela de Geociencias y Medio Ambiente, Facultad de Minas, \\ Universidad Nacional de Colombia, sede Medellín, Carrera 80 \# 65- \\ 223 Medellín,Colombie.e-mail: andres.osorio@unican.es \\ ${ }^{2}$ Grupo de Ingeniería Oceanográfica y de Costas (GIOC), Univ. \\ Cantabria, Avda Los Castros s/n, 39005, Santander, Espagne \\ ${ }^{3}$ SOGREAH Maritime, 6, rue de Lorraine, 38130 Echirolles, France \\ ${ }^{4}$ Université de Caen Basse-Normandie, Master Génie Côtier, France
}

\section{Résumé}

L'utilisation de systèmes vidéo et de procédures de traitement d'images permet de traiter concrètement un nombre croissant de problématiques d'ingénierie côtière. Le projet européen COASTVIEW (EVK3-CT-2001-0054) a permis de mettre en évidence plusieurs domaines dans lesquels les systèmes vidéo peuvent appuyer des protocoles classiques d'acquisition de données en milieu côtier et s'imposer comme des outils d'aide à la décision en matière de gestion intégrée du littoral. L'étude présentée ici, développée dans le cadre de ce projet, porte spécifiquement sur l'utilisation d'images vidéo pour l'exploitation touristique d'une plage, concrètement celle du «Puntal» (Santander, Espagne), à travers l'analyse de la distribution, spatiale et temporelle, des usagers.

\section{$\underline{\text { Abstract }}$}

The use of video imaging and images processing techniques are increasingly used worldwide to deal with a variety of coastal engineering aspects. The European project COASTVIEW (EVK3-CT-2001-0054) has emphasised various coastal engineering fields for which video systems could be used in support to more traditional procedures of data acquisition and viewed as practical tools for an integrated management. The study presented here, developed in the frame of the COASTVIEW project, specifically deals with the use of video for the survey of the recreational use of beaches and is centred, through the example of the « Puntal» beach at Santander (Spain), on the analysis of the spatial and temporal distribution of the users on the beach.

\section{$\underline{\text { Mots-clés }}$}

Système vidéo Argus, usage touristique des plages, distribution des usagers, algorithme de détection automatique, gestion intégrée du littoral. 


\section{Introduction}

Parmi les principaux problèmes auxquels se heurtent techniciens et preneurs de décisions en charge de la gestion et de l'aménagement des zones côtières, celui de la disponibilité de séries de données suffisamment longues et fiables permettant d'appuyer les options techniques et les décisions d'intervention se pose toujours avec force. Les méthodes traditionnelles d'acquisition de données de terrain ont toujours engendré d'importantes difficultés logistiques, qui freinent les progrès des connaissances sur l'environnement côtier. Des perspectives prometteuses se sont ouvertes avec le développement de techniques innovantes basées sur le traitement d'images réalisées à l'aide de caméras vidéo stratégiquement placées sur le littoral. La mise en valeur de la fiabilité, la précision et l'adaptabilité des systèmes vidéo en matière de Gestion Intégrée des Zones Côtières est l'un des objectifs du projet européen COASTVIEW (EVK3-CT-2001-0054). Le projet vise concrètement à l'établissement d'un panel de paramètres simples ("Coastal State Indicators » ou CSIs) directement liés aux enjeux essentiels de la gestion du littoral que sont la navigation, les loisirs, la protection des côtes et de l'écosystème, et au développement d'un système de caméras vidéo permettant d'obtenir une information quantitative précise de ces CSIS de la manière la plus fonctionnelle possible. Développée dans le cadre de ce projet, la présente étude s'intéresse spécifiquement à la recherche et à la mise au point d'outils et de recueils de données utiles à la gestion de l'usage touristique des plages. Cette application est traitée à travers l'exemple de la plage dite du Puntal à Santander (Espagne), choisie comme l'un des sites-pilote du projet COASTVIEW.

\section{Description du site et obtention de la base de données}

La plage du Puntal, située à l'embouchure de la baie de Santander, sur le littoral cantabrique, tient son nom de sa localisation à l'extrémité d'une flèche sableuse. Il s'agit d'une plage entièrement préservée à son état naturel et comptant parmi les plus populaires de la région. L'installation vidéo, de type Argus (Holman et al. ${ }^{1}$ ), installée par l'Université de Cantabrie pour étudier le site est constituée de quatre caméras situées sur le toit de l'hôtel Real de Santander, à une hauteur de 90 mètres et à une distance d'environ un kilomètre de la zone d'étude. Un système vidéo ARGUS type est composé de plusieurs caméras installées en hauteur et pointant vers la côte, capables d'enregistrer de façon continue des images de haute résolution. Ces images, capturées par un ordinateur et stockées localement, sont ensuite envoyées via Internet à un serveur qui en garde une copie. Les images sont finalement publiées sur Internet, afin de faciliter leur accès et leur traitement postérieur. Le système prend des images de $1024 \times 768$ pixels sur la bande du spectre visible (RGB). Chaque pixel est associé à une position sur l'image (U, V) directement en relation avec les coordonnées réelles $(\mathrm{X}, \mathrm{Y}, \mathrm{Z})$ de la zone d'étude. Une batterie de caméras pointant en direction de la zone d'étude permet d'obtenir des images pouvant être combinées pour former une vue panoramique de l'ensemble de la zone. Les images obliques provenant des différentes caméras sont généralement rectifiées et associées digitalement pour générer une vue plane 
sans distorsion de la zone. Parmi les divers types de photos disponibles, celles utilisées pour cette étude sont des images instantanées, dites de type snap. La Figure 1 montre une image de haute résolution $(2 \mathrm{~m} /$ pixel en transversal, $1.2 \mathrm{~m} /$ pixel en longitudinal), prise par l'une des caméras du système Argus de Santander. Les données utilisées pour cette étude ont été collectées entre le 15 juin et le 15 septembre 2003, à raison d'une photo par demi-heure.

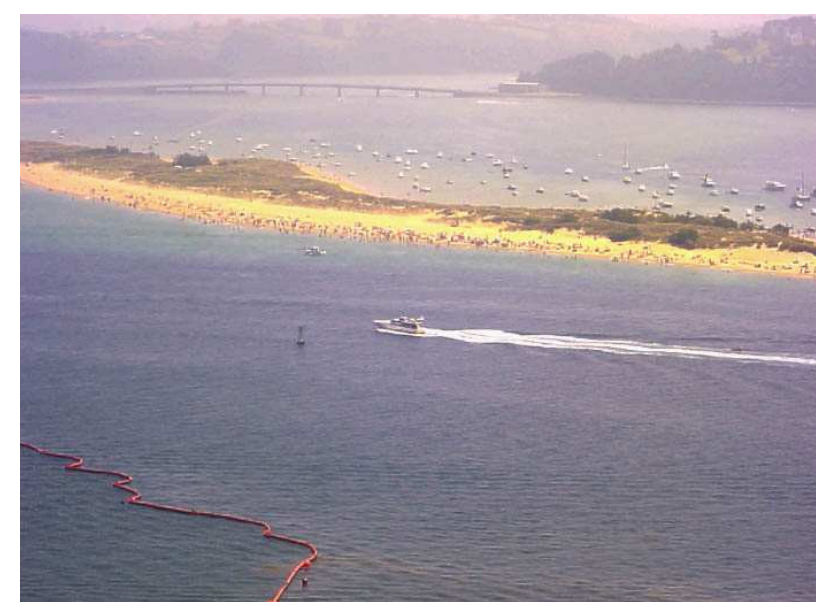

Figure 1 : Image de type « snap » (instantanée) prise par le système Argus de la plage du « Puntal » à Santander (Espagne), le 09 Août 2003 à 12:40.

\section{$\underline{\text { 3. Définition de concepts et analyse de variables }}$}

\subsection{Problématique de gestion}

Quand une plage est exploitée à des fins touristiques, les autorités chargées de sa gestion se doivent de proposer et d'administrer correctement les ressources naturelles, les services et la sécurité de celle-ci (Jiménez et $a l^{2}{ }^{2}$ ). La définition d'un plan de gestion nécessite de pouvoir évaluer le niveau d'usage de la plage à un instant donné et de définir des stratégies pour maintenir et améliorer cet usage. Chacune de ces étapes soulève la question du potentiel d'usage de la plage. Le concept de potentiel d'usage ou de capacité d'accueil d'une plage («Beach Recreational Carrying Capacity » ou BRCC), thème de recherche commun aux chargés de gestion des plages et aux scientifiques, se réfère à la quantité et au type d'usagers que peut supporter une plage sans que se produise un impact inacceptable tant au niveau environnemental que social (Manning et Lawson ${ }^{3}$ ). Le concept de BRCC est ample et complexe. L'étude présentée ici est centrée sur l'un des aspects les plus importants en relation avec cet indicateur, le niveau d'usage de la plage, associé au nombre d'usagers sur la plage et à leur position dans la zone à chaque instant, autrement dit le comportement spatial et temporel des usagers de la plage. Il est possible de réaliser un suivi de ces paramètres via des techniques traditionnelles, comme le comptage manuel ou la prise de photos en certains instants particuliers (e.g. Polette et Raucci ${ }^{4}$ ), mais ces techniques peuvent s'avérer ardues et comportent deux inconvénients: elles n'offrent pas la 
possibilité de positionner les usagers dans un système de coordonnées réelles et elles ne permettent pas un suivi continu. La première difficulté peut être compensée par l'utilisation de photographies aériennes (Pereira da Silva ${ }^{5}$ ) et la seconde par l'intermédiaire de webcams (Kammler et Schernewski ${ }^{6}$ ). Mais aucun de ces systèmes ne permet d'obtenir chacun de ces paramètres de façon continue, géoréférencée et avec un haut degré de fiabilité. Avec l'expérience acquise dans l'utilisation de systèmes vidéo géoréférencés de haute résolution comme Argus, pour des problèmes hydrodynamiques, morphodynamiques et liés à la gestion du littoral, est apparue l'idée d'utiliser les images collectées pour déterminer la distribution des usagers sur les plages. Les paragraphes suivants décrivent l'application d'une méthodologie et d'un modèle appelé BUDA (Osorio ${ }^{7}$ ) permettant, à partir d'une base de données d'images Argus, d'estimer le nombre d'usagers présents sur la plage et les positions associées à chacun d'entre eux sur chaque image et une période d'étude donnée.

\subsection{Description sommaire de l'algorithme d'acquisition des données}

L'algorithme BUDA (Beach User Detection algorithm), conçu pour identifier la position des usagers sur les plages, est un algorithme de type kernel qui s'applique sur des images de haute résolution, permettant d'associer les pixels de l'image à des personnes présentes sur la plage. Le principe de la détection est illustré Figure 2. Une zone d'intérêt (ROI, Region Of Interest), dont les dimensions varient selon l'image, comprenant tout ou partie de la plage sèche, doit être définie, afin de restreindre la zone de recherche à l'espace où se situent les vacanciers. Une personne sur la zone ROI est considérée comme un objet (pixel ou groupe de pixels) si ses propriétés sont suffisamment différentes des pixels qui l'entourent. Les paramètres de calibration de BUDA, déterminant la qualité de la détection, sont la taille du kernel idéal et le niveau d'intensité de couleur permettant d'identifier une personne sur la plage.

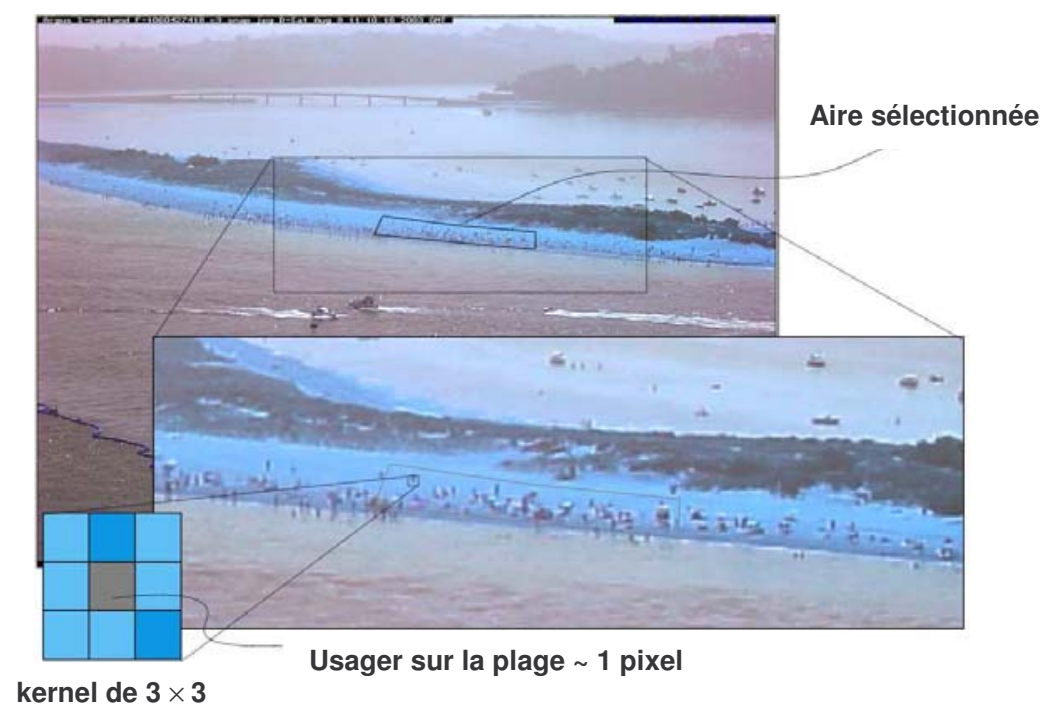

Figure 2 : Zone sélectionnée sur l'image complète et kernel de $3 \times 3$ positionné sur un groupe de pixels. 
Préalablement à son application, l'algorithme a été calibré par comparaison avec le pointage manuel des images des mois de juillet et août 2003, puis validé sur le reste des images de l'échantillon. La Figure 3 montre une comparaison des résultats obtenus sur l'échantillon de validation entre les données relevées manuellement et la détection automatique. Les résultats délivrés en sortie par l'algorithme BUDA sont normalisés et automatiquement stockés dans une base de données. Ils se présentent, pour chaque image traitée, sous la forme d'une matrice contenant les coordonnées sur l'image $(\mathrm{U}, \mathrm{V})$ et les coordonnées réelles $(\mathrm{X}, \mathrm{Y}, \mathrm{Z})$ de chaque usager pointé. De plus, le nom de chaque fichier indique les données précises de la photo traitée. La base de données obtenue est ainsi référencée dans le temps, permettant une consultation et un traitement des informations aisés et fonctionnels.

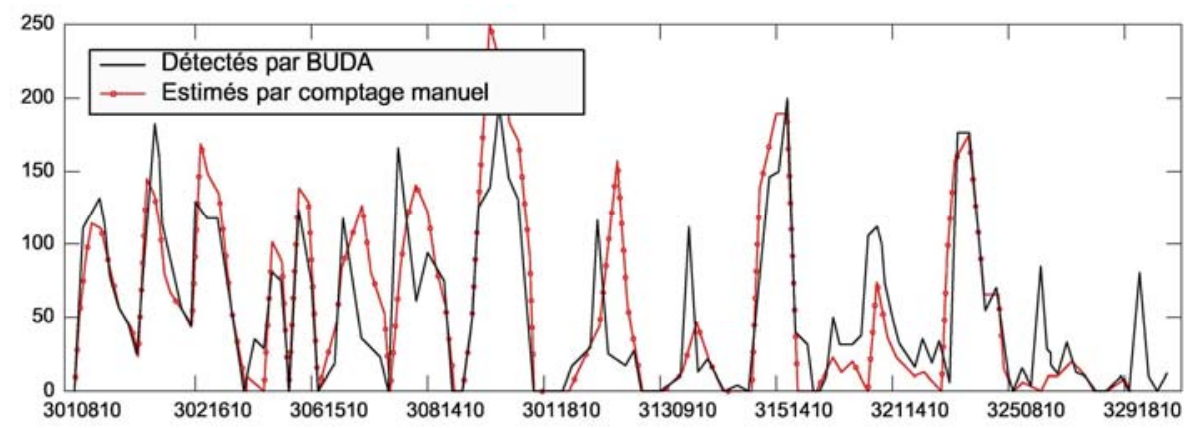

Figure 3. Comparaison des nombres d'usagers sur la plage évalués par comptage manuel et détection automatique. En ordonnées : nombre d'usagers, en abscisses : référence CDDHHMN de l'image (Caméra, Jour, Heure, Minute).

\subsection{Sélection de variables et analyse statistique}

Comme mentionné plus haut, la gestion touristique des plages requiert la connaissance de la distribution des usagers du site, évoluant dans le temps à différentes échelles (jour, semaine, saison, année) et influencée dans l'espace par les pôles d'attraction et de répulsion existant sur la plage. Parmi ces pôles, les accès à la plage, dont les usagers tendent à s'éloigner, et le rivage, dont les usagers cherchent à se rapprocher, déterminent pour une large part la distribution sur le site. Deux variables sont étudiées ici : la distance parcourue par chaque usager depuis le point d'entrée jusqu'à sa position sur la plage sèche et la distance à laquelle l'usager se situe par rapport au trait de côte. Le calcul de ces variables suppose de pouvoir déterminer le positionnement des usagers dans un repère local géoréférencé, ce qui a été réalisé via l'exploitation des caméras Argus et de la base de données générée par l'application de l'algorithme BUDA. L'analyse présentée ici utilise des algorithmes spécialement développés pour l'exploitation et l'analyse de la base de données générée par BUDA. La plage du Puntal se prête particulièrement bien à l'analyse des deux variables citées : il s'agit en effet d'une plage naturelle, où aucun des services disponibles sur une plage urbaine n'est proposé et où le seul pôle d'attraction est donc la proximité du rivage. Par ailleurs, bien qu'il soit possible d'accéder à la plage à pied, en longeant la flèche sableuse 
sur $3 \mathrm{~km}$ jusqu'à la pointe, la plupart des estivants y accèdent au moyen d'un ferry connectant la ville de Santander au ponton situé à l'extrémité de la flèche. Cette configuration fait de cette plage un excellent site dédié à l'étude de la distribution spatiale et temporelle des usagers, dans le sens où l'on peut estimer que tous les vacanciers proviennent de cet unique point d'entrée.

La Figure 4 présente un exemple des résultats obtenus par une analyse statistique de la variable « distance à l'accès ». La figure présente l'évolution temporelle de la distribution des distances parcourues (en haut à gauche), l'évolution des distances moyennes et des écart-types (au milieu à gauche), l'évolution de la fréquentation (en bas à gauche) et la distribution des distances parcourues (à droite). Une analyse similaire est réalisée avec la variable « distance au rivage » avec comme ligne de côte de référence le niveau de basse-mer de vives-eaux. Les deux variables suivent (à 98\%) une loi de distribution log-normale, comme le montre l'histogramme. La moyenne et la déviation des données de l'échantillon suffisent donc à décrire le comportement des usagers en termes de distance au point d'entrée sur la plage et au rivage. L'hypothèse selon laquelle tous les usagers de la plage du Puntal entrent sur la plage par un accès unique permet d'établir le comportement spatial et temporel suivant: les usagers marchent sur une distance d'entre 70 et $200 \mathrm{~m}$ depuis l'accès, avec une distance optimale de $100 \mathrm{~m}$ et une proximité au rivage évaluée par ailleurs entre 10 et $30 \mathrm{~m}$. Les paramètres statistiques décrivant les deux variables sont constants au cours du temps, suggérant que la distribution des personnes sur la plage ne dépend pas du nombre d'usagers et est déterminée principalement par les pôles d'attraction et de répulsion, comme les accès, le rivage et la position optimale sur la plage.

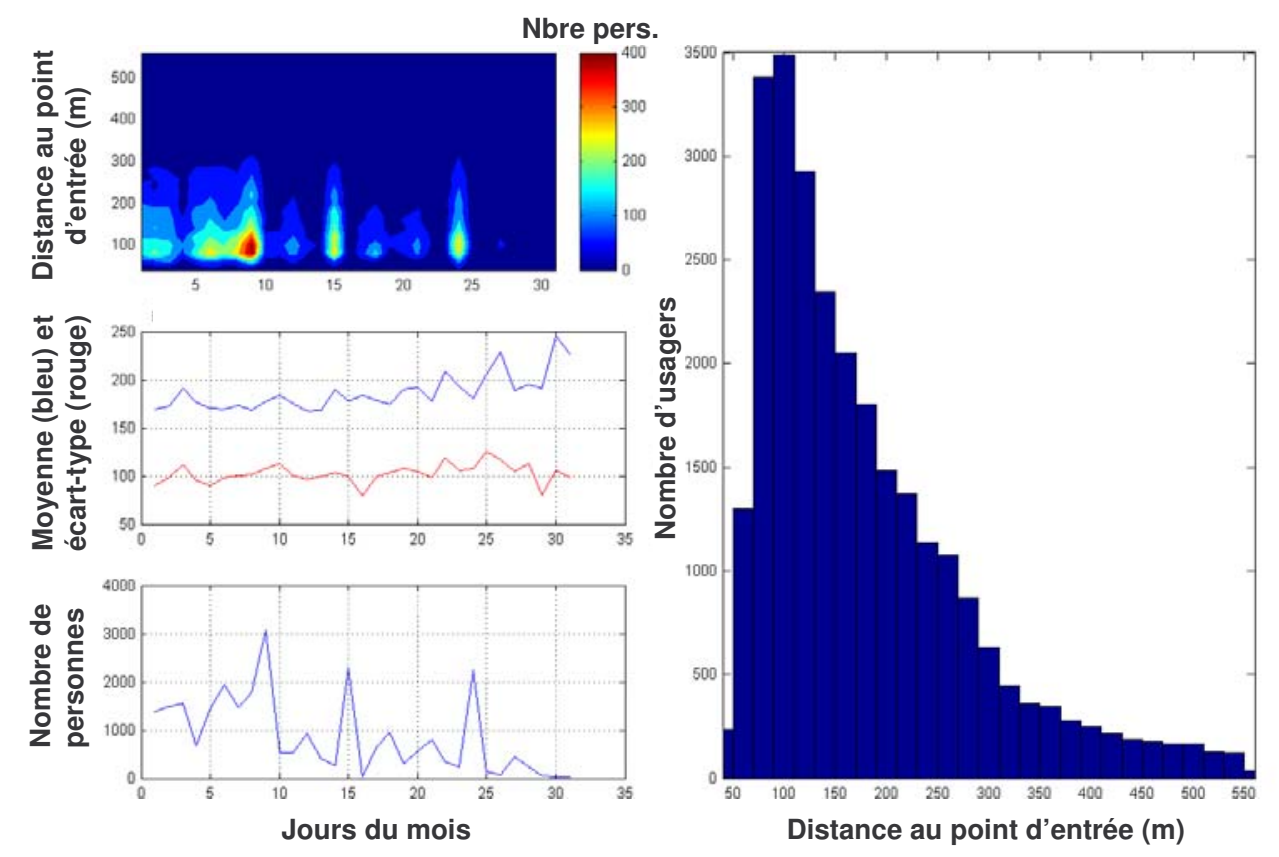

Figure 4 : Distribution des usagers de la plage du Puntal par rapport au point d'entrée (résultats du mois d'août 2003). 
Une troisième variable, la densité d'occupation de la plage, est analysée. Ce concept peut être associé au nombre de personnes par mètre carré ou à la surface de plage disponible pour chaque usager. Cette dernière définition est très utilisée dans la littérature pour évaluer le niveau de saturation d'une plage (e.g. De Ruyck et al., 1970). Pour l'estimation de cette variable sur la plage du Puntal, une maille rectangulaire de $50 \mathrm{~m} \times 50 \mathrm{~m}$ a été établie. Les densités moyennes mensuelles, moyennes journalières et maximales journalières ont été étudiées. A titre d'exemple, la Figure 5 montre le niveau d'occupation moyen mensuel de la plage, calculé avec une maille plus fine de $20 \mathrm{~m} \times 20 \mathrm{~m}$, avec un pic d'occupation au point de coordonnées $(-300 ; 60)$. Le même résultat est obtenu pour la densité moyenne journalière. L'analyse montre que le même comportement est observé en termes de densité maximale.

L'analyse de la densité d'occupation de la plage montre donc un pic (position optimale sur la plage) constant dans le temps et l'espace. Ce point est directement lié aux pôles d'attraction/répulsion évoqués plus haut, c'est-à-dire à la distance que l'usager est disposé à parcourir pour se trouver en première ligne de plage. Par ailleurs, il a été observé que la distribution des usagers est asymétrique par rapport à l'accès, avec une concentration dans la zone Est de la plage. Ceci s'explique par la position de l'accès à l'Ouest et l'impact visuel provoqué dans cette zone par l'activité portuaire, le chenal d'accès au port passant très près de la pointe de la flèche qui constitue la partie occidentale de la plage.

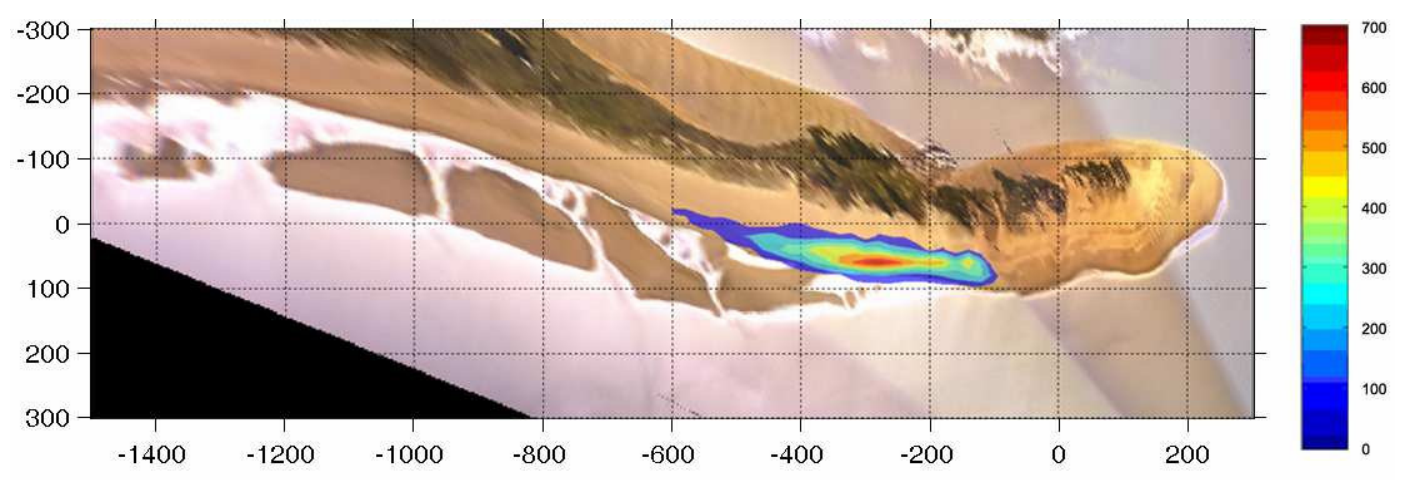

Figure 5 : Densité moyenne mensuelle pour le mois d'août, en nombre d'usagers par cellule de 20 $\times 20 \mathrm{~m}^{2}$ (images assemblées et rectifiées).

\section{$\underline{\text { 4. Discussion et conclusions }}$}

L'analyse statistique montre que les variables étudiées (distance parcourue depuis l'accès à la plage et distance au rivage) suivent des fonctions de distribution lognormale, que la variation des paramètres statistiques est uniforme, que les fonctions de densité présentent les mêmes tendances et que les pics d'occupation maximale coïncident dans l'espace, indépendamment de l'heure, du jour ou du mois. Cette information permet d'établir, à partir d'une partie de l'échantillon de données, un modèle probabiliste pour pronostiquer la distribution des usagers sur 
la plage, basée sur une matrice de probabilité d'occupation d'un endroit donné de la plage. L'erreur entre la distribution d'usagers évaluée automatiquement et celle calculée par le modèle probabiliste est de 5 à $15 \%$ sur l'ensemble de l'échantillon. Il a par ailleurs été montré que les modèles paramétriques de densité maximale et probabiliste de distribution permettent de prédire le niveau d'usage à n'importe quelle heure, jour et époque de l'année en fonction du nombre de visiteurs attendus. En d'autres termes, les modèles proposés servent à connaître les points d'occupation maximale de la plage, le niveau de saturation et la distribution des utilisateurs. Ces modèles, couplées à l'obtention des données fournies par l'algorithme BUDA et l'application d'une méthodologie pour analyser le niveau d'usage de la plage, sont de grande utilité pour déterminer le type d'usage de la plage, le comportement des usagers, le niveau de saturation de certaines zones de la plage, l'efficacité et la bonne utilisation des points d'entrée, etc. Ils apportent ainsi des informations permettant d'aider les institutions chargées de la gestion des plages à définir et/ou optimiser des projets d'aménagement ou de restauration des plages.

\section{Références}

1 Holman, R.A., Sallenger Jr, A.H., Lippmann, T.C. and Haines, J.W. (1993), The application of video image processing to the study of nearshore processes. Oceanography, 6 (3), 78-85.

2 Jiménez, J.A., Osorio, A., Marino-Tapia, I., Kroon, A., Medina, R., Davidson, M., Archetti, R., Ciavola, P., Aarninkhof, S. (2005), Beach recreation planning using video-derived coastal state indicators. Coastal Engineering, Special Issue of the CoastView project. Sous presse.

3 Kammler, M., Schernewski, G. (2004), Spatial and temporal analysis of beach tourism using webcam and aerial photographs, Managing the Baltic Sea. Coastline Reports 2, S. 121 - 128, ISSN 0928-2734.

4 Manning, R.E., Lawson, S.R. (2002), Carrying capacity as "informed judgment": the values of science and the science of values. Environmental Management, 30, 157-168.

5 Osorio, A.F. (2005), Desarrollo de técnicas y metodologías basadas en sistemas de vídeo para la gestión de la costa. Tesis Doctoral, Universidad de Cantabria, España.

6 Pereria da Silva, C. (2002), Beach Carrying Capacity Assessment: How important is it? Journal of Coastal Research, Special Issue 36, 190-197.

7 Polette, M., Raucci, G.D. (2003), Methodological proposal for carrying capacity analysis in sandy beaches: a case study at the Central beach of Balneario Camboriú (Santa Catarina, Brazil). Journal of Coastal Research, Special Issue 35, 94-106. 